\title{
Produtividade de colmos em clones de cana-de-açúcar ${ }^{1}$
}

\author{
Katiule Pereira Morais ${ }^{2 *}$, Sandro Luis Petter Medeiros ${ }^{3}$, Sergio Delmar dos Anjos e Silva , Jean Cecchin Biondo ${ }^{5}$, \\ Jessica Hoch Boelter ${ }^{6}$, Fagner Souto Dias ${ }^{7}$ \\ $10.1590 / 0034-737 X 201764030010$
}

\section{RESUMO}

O objetivo deste trabalho foi avaliar a produtividade e as características de colmos de 25 clones de cana-de-açúcar, nos cultivos de cana-planta e cana-soca. O experimento foi realizado nos anos agrícolas de 2009-2010 (cana-planta) e 2010-2011 (cana-soca), em Jaguari, RS. O delineamento utilizado foi o de blocos ao acaso, em esquema fatorial (clones e cultivos de cana-planta e cana-soca), com três repetições. Ao final de cada cultivo, foram realizadas avaliações de comprimento, diâmetro, número e produtividade de colmos. Os dados obtidos foram submetidos à análise da variância e, as médias, comparadas pelo teste de Tukey, a 5\% de probabilidade A produtividade média de colmos dos clones mais produtivos de ciclo precoce, nos cultivos de cana-planta e cana-soca (96,6 e 123,4 tha ${ }^{-1}$, respectivamente) foi semelhante à obtida com os clones mais produtivos de ciclo médio-tardio (101,5 e 128,0 t ha-1 , respectivamente). No cultivo de cana-soca, a produtividade média de colmos superou a obtida no cultivo de cana-planta, com 13 dos 25 clones avaliados. Em geral, os clones mais produtivos apresentam valores elevados de número e de comprimento de colmos, sendo observada baixa variação do diâmetro de colmos dos materiais testados. Os clones RB965911, RB925345, RB855156, RB987935, RB935744, RB867515, RB975019, RB925268, RB845210 e RB975329 são os mais adaptados às condições edafoclimáticas da região da Depressão Central do Rio Grande do Sul, apresentando maior produtividade de colmos, com valores acumulados, nos dois cultivos, superiores a $200 \mathrm{t} \mathrm{ha}^{-1}$.

Palavras-chave: Saccharum sp.; parâmetros de colmo; produção.

\section{ABSTRACT}

\section{Productivity and agronomic variables in sugarcane clones}

The objective of this study was to evaluate the productivity and characteristic of stalks from 25 sugarcane clones in plant-cane and ratoon cultivations. The experiment was conducted in Jaguari, RS, Brazil, during the 2009/2010 (plant cane) and 2010/2011 (ratoon) growing seasons. The experimental design was randomized blocks in a $25 \times 2$ factorial arrangement (25 clones and two growing seasons), with three replications. At the end of each growing season, the length, diameter, and number and productivity of stalks were evaluated. The data were subjected to analysis of variance and means were compared by Tukey test at $5 \%$ probability. The average stalk yield of in the most productive clones with early cycle in plant-cane and ratoon (96.6 and $123.4 \mathrm{t} \mathrm{ha}^{-1}$, respectively) was similar to that obtained with the most productive clones with mid-late cycle (101.5 and $128 \mathrm{t} \mathrm{ha}^{-1}$, respectively). The stalk yield in ratoon exceeded that obtained in the plant-cane in 13 of the 25 clones evaluated. In general, the most productive clones have elevated number and length of stalks, with an observed low variation in stalk diameter of the tested materials. The clones RB965911, RB925345, RB855156, RB987935, RB935744, RB867515, RB975019, RB925268, RB845210, and RB975329 are the most adapted to the soil and climatic conditions of the Depressão Central do Rio Grande do Sul region, with higher stalk yield, with values accumulated in the two growing seasons above $200 \mathrm{t} \mathrm{ha}^{-1}$.

Key words: culm parameters; production; Saccharum sp.

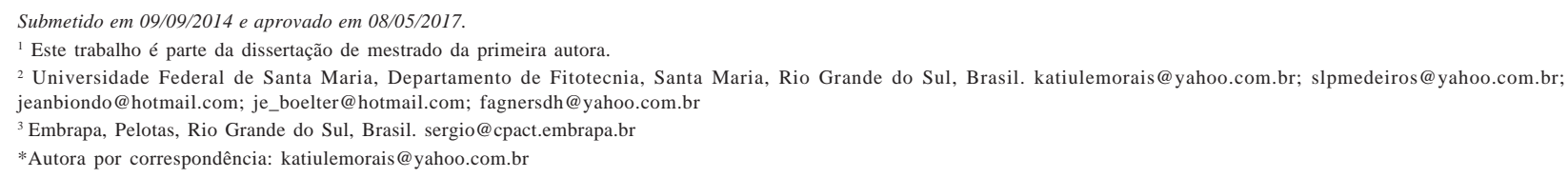




\section{INTRODUÇÃO}

Mundialmente, o Brasil destaca-se com a cultura da cana-de-açúcar para a produção de açúcar e como fonte de energia renovável. No Estado do Rio Grande do Sul (RS), a área plantada, destinada a produção sucroalcooleira, na safra 2009-2010, foi de 35.970 ha, com rendimento médio de $41,8 \mathrm{t} \mathrm{ha}^{-1}$, muito aquém do rendimento de outros Estados produtores e da produtividade média nacional de $81 \mathrm{t}$ ha $^{-1}$ (Conab, 2012). Isso se deve, principalmente, à carência de clones modernos, com maiores potenciais de produção e adaptados às condições agrometeorológicas do Rio Grande do Sul.

Ao avaliarem clones de cana-de-açúcar, em Cruz Alta, RS, Canal \& Matzenbacher (1986) concluíram que a ocorrência de baixas temperaturas do ar foi o fator limitante ao cultivo, na região, pois nenhum material testado foi tolerante ao frio. Porém, Aude et al. (1994), estudando adaptação de clones de cana-de-açúcar, em Santa Maria, RS, constataram que nove dos dez clones testados poderiam ser recomendados para a região, pois apresentaram alta produção de colmos industrializáveis e elevados teores de sólidos solúveis no caldo. Acrescentaram, ainda, ser possível realizar até quatro cortes, visto que somente ocorreu decréscimo acentuado da produtividade de colmos a partir da quinta colheita.

Fatores genéticos relacionados com clones definem o número de colmos por planta, a altura e o diâmetro do colmo, o comprimento e a largura das folhas e a arquitetura da parte aérea, sendo a expressão desses caracteres muito influenciada por clima, manejo e práticas culturais utilizadas (Rodrigues, 1995). O comprimento do colmo aumenta quando o comprimento do dia varia entre 10 e 14 horas (Barbieri \& Vila Nova, 1981), condição essa encontrada nos meses de primavera-verão no Rio Grande do Sul. Além disso, a disponibilidade de radiação solar também é elevada, proporcionando, portanto, condições meteorológicas favoráveis à cultura da cana-de-açúcar (Cargnelutti Filho et al., 2004).

Cerca de dez milhões de litros de etanol, por ano, são produzidos no Rio Grande do Sul, o que representa apenas $2 \%$ de todo o consumo do Estado. A grande parte do combustível utilizado pelo Estado é comprada do centro do país, o que aumenta o custo logístico. Para buscar soluções, produtores, entidades de pesquisa e revendedores querem viabilizar a cadeia produtiva no Rio Grande do Sul. O primeiro passo parte da pesquisa, que trabalha na adaptação da cana-de-açúcar ao clima gaúcho (Fepagro, 2012).

A identificação de clones mais adaptados para as condições edafoclimáticas do Rio Grande do Sul é um fator crucial para produção de cana-de-açúcar por unidade de área, permitindo elevar a rentabilidade e, assim, a sustentabilidade da propriedade rural, além de favorecer a eficiência da ca- deia produtiva do álcool no Estado. Por essas razões, este trabalho tem por objetivo estudar o desempenho agronômico e os atributos de colmos de 25 clones de cana-de-açúcar, em dois cultivos (cana-planta e cana-soca) no município de Jaguari, Estado do Rio Grande do Sul.

\section{MATERIAL E MÉTODOS}

O experimento foi conduzido na área experimental do Núcleo de Pesquisa Avançada, do Instituto Federal de Educação, Ciência e Tecnologia Farroupilha (IFET), sediado na localidade do Chapadão, município de Jaguari, RS, Brasil (29²9' S, 5441'O), de julho de 2009 a julho de 2011 . O clima da região é o Cfa (subtropical úmido, com verões quentes e sem estação seca definida), conforme classificação de Köppen (Moreno, 1961).

Os tratamentos foram organizados no delineamento experimental em blocos ao acaso, em esquema fatorial (clones e cultivos de cana-planta e cana-soca), com três repetições, totalizando 75 unidades experimentais. Os clones avaliados foram desenvolvidos pela Rede Interuniversitária para o Desenvolvimento do Setor Sucroalcooleiro (RIDESA), sendo 12 de ciclo precoce (RB835054, RB855156, RB925211, RB925345, RB965902, RB965911, RB966923, RB935581, RB975932, RB986419, RB986955 e RB996961)e 13 de ciclo médio-tardio (RB72454, RB845210, RB867515, RB935744, RB925268, RB966229, RB975038, RB975329, RB975019, RB945177, RB947625, RB008347 e RB987935). Esses 25 clones foram provenientes da Embrapa Clima Temperado, onde foram selecionados pelo melhor desempenho a partir de 224 materiais testados em dois anos de seleção. Neste estudo, os 25 clones foram avaliados nos cultivos de cana-planta (2009-2010) e cana-soca (2010-2011).

Antes da implantação do experimento, a área experimental recebeu uma aração, seguida de gradagem e aplicação de $3 \mathrm{t} \mathrm{ha}^{-1}$ de calcário. O plantio foi realizado em sulcos, na densidade de 18 gemas por metro linear, espaçamento entre linhas de 1,4 m e profundidade de plantio de $20 \mathrm{~cm}$. No momento do plantio (01/09/2009), foi efetuada a adubação de correção de P, K e micronutrientes, de acordo com o resultado da análise de solo e o recomendado pela CQFS - RS/SC (2004). Para isso, foram aplicados $480 \mathrm{~kg}$ ha${ }^{1}$ da fórmula 0-25-20 e $30 \mathrm{~kg} \mathrm{ha}^{-1}$ de FTE BR12 (4\% $\mathrm{K}_{2} \mathrm{O}$; $1,8 \% \mathrm{~B} ; 0,8 \% \mathrm{Cu} ; 3 \% \mathrm{Fe} ; 2 \% \mathrm{Mn} ; 2 \% \mathrm{Mo} ; 9 \% \mathrm{Zn})$. A adubação de $\mathrm{N}$ em cobertura foi realizada com ureia, com a dose de $120 \mathrm{~kg} \mathrm{ha}^{-1}$, dividida em duas aplicações: a primeira, no dia 23 de novembro de 2009 e, a segunda, no dia 17 de janeiro de 2010, aos 85 e 141 dias após o plantio, respectivamente. A adubação da soqueira foi realizada no dia 7 de setembro de 2010, com as aplicações de $60 \mathrm{~kg} \mathrm{ha}^{-1}$ de $\mathrm{KCl}$ e de $60 \mathrm{~kg} \mathrm{ha}^{-1}$ de $\mathrm{P}_{2} \mathrm{O}_{5}$. A adubação nitrogenada em cobertura foi a mesma utilizada em cana-planta, aplicada 
nos dias 01 de novembro de 2010 e 15 de janeiro de 2011 . No início, foi realizada capina manual e, no decorrer do ciclo, sempre que necessário, foram aplicados os herbicidas Gramoxone e 2,4 D na entrelinha da cultura. O controle da broca-da-cana-de-açúcar (Diatraea saccharalis) foi realizada por predação, através da vespa Cotesia flavipes.

A colheita da cana-planta foi realizada em 30 de julho de 2010. A brotação da soqueira ocorreu em setembro de 2010 e a colheita da cana-soca em julho de 2011. Nos dois cultivos, a colheita das plantas das parcelas foi realizada manualmente, efetuando-se um corte na base dos colmos de toda a linha central da parcela $(5 \mathrm{~m})$, sendo, posteriormente, pesados em balança de capacidade para $100 \mathrm{~kg}$, instalada em tripé, para a avaliação de produtividade de colmos comerciais. No momento da colheita, também foram avaliados os seguintes atributos dos colmos: a) diâmetro de colmo (DC) - medido com paquímetro no terço inferior de dez colmos por bloco; b) comprimento de colmo (CC) - medido pelo comprimento entre o ponto de corte e o ponto de quebra do palmito de dez colmos por bloco; c) número de colmos - obtido pela contagem de colmos por metro ( $\mathrm{NC}$ metro ${ }^{-1}$ ).

Os dados obtidos foram submetidos à análise da variância e, as médias, comparadas pelo teste de Scott Knott, a 5\% de probabilidade.

\section{RESULTADOS E DISCUSSÃO}

\section{Número, diâmetro e comprimento de colmos}

Nos estudos com os clones de ciclo precoce, não se verificou interação com os cultivos para o número de colmos $\mathrm{m}^{-1} \mathrm{e}$ para o diâmetro de colmo (Tabela 1). Além disso, não foram observadas diferenças marcantes quanto ao número e ao diâmetro do colmo entre os clones, sendo observados somente dois grupos que se diferenciaram entre si, em cada variável, e outro grande grupo intermediário, com desempenhos estatisticamente semelhantes. Os clones RB855156, RB925345 e RB965911 somente diferenciaram-se estatisticamente quanto ao número de colmos $\mathrm{m}^{-1}$, em relação ao clone RB986419, porém este não diferiu estatisticamente dos demais (Tabela 2). Para o diâmetro de colmo, o clone RB986419 somente apresentou diferença estatística com RB925345, RB835054, RB965902 e RB855156. Assim, constata-se que, tanto para número quanto para comprimento de colmos, não existe uma superioridade evidente para um clone ou mesmo grupo.

Quanto à variável comprimento de colmo (Tabela 2) foi constatada interação dos clones de ciclo precoce com os cultivos, os quais mantiveram ou aumentaram o comprimento do colmo da cana-planta para a soca. Hanauer (2011), para os clones SP71406 e CB4176, em Santa Maria, RS, também encontrou valores de comprimento de colmo superiores em cana-soca, corroborando os resultados encontrados neste estudo, para os clones de ciclo precoce, em Jaguari, RS. Analisando-se, porém, os cultivos, não foi verificada grande diferenciação quanto ao comprimento do colmo entre os clones, na cana-planta e na cana-soca, pois, de 12 clones, dez foram estatisticamente semelhantes entre si, dentro de cada sistema.

Foi observada interação entre os clones de ciclo médio-tardio e os cultivos, para o número de colmos (Tabela 3), os quais geralmente mantiveram ou diminuíram o número, da cana-planta para a cana-soca. Não foi verificada superioridade de clones com relação ao número de colmos, pois as respostas foram muito similares, visto que houve um grande agrupamento de clones que não apresentaram diferenças estatísticas entre si, principalmente, em canaplanta e, em menor grau, em cana-soca. O clone RB975038 apresentou número de colmos superior em cana-planta, porém diferiu estatisticamente apenas dos clones RB947625, RB945177, RB008347 e RB975329. Em cana-soca, os clones RB935744 e RB975019 apresentaram diferença significativa apenas para os RB867515 e RB947625. Observou-se variação do desempenho dos clones, quanto ao número de colmos, nos dois sistemas de cultivo, citando-se, por exemplo, RB945177, RB008347 e RB975329, que apresenta-

Tabela 1: Análise da variância realizada para as variáveis Produtividade, Número de colmos por metro $\left(\mathrm{NC}^{-1}\right)$, Diâmetro de colmos (DC) e Comprimento de colmos (CC), de clones de cana-de-açúcar de ciclos precoce e médio-tardio, de cana-planta e cana-soca

\begin{tabular}{|c|c|c|c|c|c|}
\hline & \multicolumn{5}{|c|}{ Ciclo Precoce } \\
\hline & GL & Produtividade & $\mathrm{NC} \mathrm{m}^{-1}$ & DC & $\mathrm{CC}$ \\
\hline Clones & 1 & $96,92 *$ & $93,61^{*}$ & $57,73^{*}$ & $93,37 *$ \\
\hline Ciclo & 11 & $18,23^{*}$ & $4,68^{\mathrm{ns}}$ & $1,88^{\mathrm{ns}}$ & $7,78^{*}$ \\
\hline Clones X Ciclo & 11 & $6,23 *$ & $1,63^{\mathrm{ns}}$ & $0,87^{\mathrm{ns}}$ & $3,57^{\mathrm{ns}}$ \\
\hline \multicolumn{6}{|c|}{ Ciclo Médio-Tardio } \\
\hline & GL & Produtividade & $\mathrm{NC} \mathrm{m^{-1 }}$ & DC & $\mathrm{CC}$ \\
\hline Clones & 1 & $81,26^{*}$ & $16,4^{*}$ & $59,52 *$ & $93,61 *$ \\
\hline Ciclo & 12 & $10,61 *$ & $10,21 *$ & $2,38^{\mathrm{ns}}$ & 4,68 ns \\
\hline Clones X Ciclo & 12 & $7,13^{*}$ & $5,67 *$ & $3,43^{\mathrm{ns}}$ & $1,63^{\mathrm{ns}}$ \\
\hline
\end{tabular}

*significativo ${ }^{\text {ns }}$ não significativo a $5 \%$ de probabilidade 
ram menor valor de número de colmos na cana-planta e maior valor em cana-soca.

Não foram verificadas diferenças estatísticas entre os clones de ciclo médio-tardio, quanto ao diâmetro do colmo (Tabela 3), as quais somente ocorreram para o comprimento do colmo; porém as respostas foram similares, pois não houve destaque de um grupo ou mesmo de um clone. Exemplifica-se, pela resposta do RB867515, que, apesar do maior comprimento de colmo, não apresentou diferença significativa entre este e outros cinco clones, porém diferindo estatisticamente de outros sete. Entre 13 testados, 11 clones apresentaram-se estatisticamente semelhantes. A importância da caracterização do compri-

Tabela 2: Número de colmos por metro (NC/metro), Diâmetro de colmo (DC) e Comprimento de colmo (CC) de clones de ciclo precoce de cana-de-açúcar, de cana-planta e cana-soca. Jaguari, RS, 2009-2011 (1)

\begin{tabular}{|c|c|c|}
\hline Clones & $\mathrm{NC} \mathbf{m}^{-1}$ & $\mathrm{DC}(\mathrm{cm})$ \\
\hline RB925211 & $14,15 \mathrm{a}$ & $2,78 \mathrm{ab}$ \\
\hline RB925345 & $13,51 \mathrm{a}$ & $2,60 \mathrm{~b}$ \\
\hline RB965911 & $13,15 \mathrm{a}$ & $2,97 \mathrm{ab}$ \\
\hline RB835054 & $12,63 \mathrm{ab}$ & $2,57 \mathrm{~b}$ \\
\hline RB965902 & $12,32 \mathrm{ab}$ & $2,54 \mathrm{~b}$ \\
\hline RB855156 & $11,93 \mathrm{ab}$ & $2,61 b$ \\
\hline RB966923 & $11,87 \mathrm{ab}$ & $2,70 \mathrm{ab}$ \\
\hline RB986955 & $11,77 \mathrm{ab}$ & $2,92 \mathrm{ab}$ \\
\hline RB975932 & $11,67 \mathrm{ab}$ & $2,86 a b$ \\
\hline RB935581 & $11,40 \mathrm{ab}$ & $2,68 \mathrm{ab}$ \\
\hline RB996961 & $10,55 \mathrm{ab}$ & $2,93 \mathrm{ab}$ \\
\hline RB986419 & $8,87 \mathrm{~b}$ & $3,18 \mathrm{a}$ \\
\hline $\mathrm{CV}(\%)$ & 11,51 & 6,26 \\
\hline Cana-planta & $12,96 \mathrm{a}$ & $2,47 a$ \\
\hline Cana-soca & $11,03 \mathrm{~b}$ & $3,10 \mathrm{a}$ \\
\hline $\mathrm{CV}(\%)$ & 4,46 & 7,61 \\
\hline \multirow{2}{*}{ Clones } & \multicolumn{2}{|c|}{$\mathrm{CC}(\mathrm{cm})$} \\
\hline & Cana-planta & Cana-soca \\
\hline RB925211 & 168,67 abcA & $210,67 \mathrm{abcA}$ \\
\hline RB925345 & $169,00 \mathrm{abcB}$ & $235,67 \mathrm{aA}$ \\
\hline RB965911 & $152,00 \mathrm{cB}$ & $227,23 \mathrm{abA}$ \\
\hline RB835054 & $159,50 \mathrm{bcA}$ & $167,90 \mathrm{cA}$ \\
\hline RB965902 & $172,00 \mathrm{abcA}$ & 194,33 abcA \\
\hline RB855156 & $146,67 \mathrm{cB}$ & $175,10 \mathrm{bcA}$ \\
\hline RB966923 & $155,83 \mathrm{bcB}$ & $214,57 \mathrm{abcA}$ \\
\hline RB986955 & $158,67 \mathrm{bcA}$ & 175,37 bcA \\
\hline RB975932 & $201,00 \mathrm{aA}$ & $217,57 \mathrm{abcA}$ \\
\hline RB935581 & $166,17 \mathrm{abcB}$ & $191,00 \mathrm{abcA}$ \\
\hline RB996961 & $191,00 \mathrm{abA}$ & $200,67 \mathrm{abcA}$ \\
\hline RB986419 & $144,33 \mathrm{cB}$ & $173,23 \mathrm{bcA}$ \\
\hline Média & 165,40 & 198,61 \\
\hline $\mathrm{CV}(\%)$ & 7,52 & 9,61 \\
\hline
\end{tabular}

mento do colmo é relatada por Ferreira et al. (2007), os quais citam que essa variável é a que melhor se correlaciona com a produtividade.

Dentre as variáveis relacionadas com a caracterização do colmo, o diâmetro de colmos foi aquele que apresentou menor variabilidade, tanto entre os clones de ciclo precoce (Tabela 2) quanto os de ciclo médio-tardio (Tabela 3). Resposta similar também foi encontrada por Leite et al. (2010), que, em trabalho realizado em Tocantins, também não verificaram diferença para essa variável entre os clones RB835486, RB855536, RB855113, SP791911 e IAC865480.

Tabela 3: Número de colmos por metro (NC/metro), Diâmetro de colmo (DC) e Comprimento de colmo (CC) de clones de canade-açúcar de ciclo médio-tardio, de cana-planta (2009-2010) e cana-soca (2010-2011). Jaguari, RS, 2009-2011(1)

\begin{tabular}{|c|c|c|}
\hline Clones & DC (cm) & $(\mathrm{cm})$ \\
\hline RB975038 & $2,63 \mathrm{a}$ & $188,89 \mathrm{abc}$ \\
\hline RB975019 & $2,97 \mathrm{a}$ & $182,33 \mathrm{bc}$ \\
\hline RB987935 & $2,89 \mathrm{a}$ & $188,78 \mathrm{bc}$ \\
\hline RB72454 & $3,12 \mathrm{a}$ & $176,94 \mathrm{bc}$ \\
\hline RB845210 & $2,91 \mathrm{a}$ & $191,06 \mathrm{abc}$ \\
\hline RB925268 & $3,13 \mathrm{a}$ & $180,78 \mathrm{bc}$ \\
\hline RB867515 & $3,10 \mathrm{a}$ & $223,64 \mathrm{a}$ \\
\hline RB966229 & $2,80 \mathrm{a}$ & $183,84 \mathrm{bc}$ \\
\hline RB935744 & $2,93 \mathrm{a}$ & $196,28 \mathrm{abc}$ \\
\hline RB947625 & $3,00 \mathrm{a}$ & $167,86 \mathrm{c}$ \\
\hline RB945177 & $3,08 \mathrm{a}$ & $178,33 \mathrm{bc}$ \\
\hline RB008347 & $2,93 \mathrm{a}$ & $191,45 a b c$ \\
\hline RB975329 & $2,75 \mathrm{a}$ & $208,39 \mathrm{ab}$ \\
\hline $\mathrm{CV}(\%)$ & 7,68 & 6,16 \\
\hline Cana-planta & $2,73 \mathrm{~b}$ & $171,28 \mathrm{~b}$ \\
\hline Cana-soca & $3,15 \mathrm{a}$ & 206,96 a \\
\hline $\mathrm{CV}(\%)$ & 2,48 & 2,17 \\
\hline \multirow{2}{*}{ Clones } & \multicolumn{2}{|c|}{ NC/metro } \\
\hline & Cana-planta & Cana-soca \\
\hline RB975038 & $15,14 \mathrm{aA}$ & $10,10 \mathrm{abcB}$ \\
\hline RB975019 & $14,74 \mathrm{abA}$ & $13,56 \mathrm{aA}$ \\
\hline RB987935 & $14,39 \mathrm{abcA}$ & $13,39 \mathrm{abB}$ \\
\hline RB72454 & 14,18 abcA & 11,37 abcA \\
\hline RB845210 & 13,39 abcA & $12,06 \mathrm{abcA}$ \\
\hline RB925268 & $13,22 \mathrm{abcA}$ & $12,64 \mathrm{abA}$ \\
\hline RB867515 & 12,89 abcdA & $9,56 \mathrm{bcB}$ \\
\hline RB966229 & 12,55 bcdeA & 9,87 abcA \\
\hline RB935744 & $12,14 \mathrm{cdeA}$ & $13,69 \mathrm{aA}$ \\
\hline RB947625 & 10,70 defA & $8,41 \mathrm{cB}$ \\
\hline RB945177 & 10,37 efA & $11,11 \mathrm{abcA}$ \\
\hline RB008347 & $9,33 \mathrm{fA}$ & $10,50 \mathrm{abcA}$ \\
\hline RB975329 & $8,45 \mathrm{fA}$ & $11,59 \mathrm{abcB}$ \\
\hline Média & 12,42 & 11,37 \\
\hline $\mathrm{CV}(\%)$ & 6,11 & 11,53 \\
\hline
\end{tabular}




\section{Produtividade}

Observou-se interação, entre clones de ciclo precoce e médio-tardio, com os cultivos, para a produtividade de colmos (Tabela 1). Quanto aos clones de ciclo precoce, ocorreu maior distinção na produtividade de colmos, em cana-planta, do que em cana-soca, com maior agrupamento entre clones que não apresentaram diferenças estatísticas entre si, com destaque para os clones RB965911 e RB925345 nos dois cultivos (Tabela 4). Para os de ciclo médio-tardio, o número de clones com desempenho estatístico semelhante foi maior em cana-planta, salientandose os clones RB987935, RB935744, RB867515, RB975019e RB925268, independentemente do sistema de cultivo.

Os clones RB925345 e RB965911 fizeram parte do grupo dos mais produtivos nos dois cultivos (Tabela 4) e, segundo Hoffmann (2008), o clone RB925345 pertence ao grupo dos vinte materiais mais utilizados no Brasil. Possivelmente, a grande utilização do RB925345 esteja relacionada com a elevada produção de colmos, conforme foi verificado neste trabalho, evidenciando também as condições agro-climáticas favoráveis para implantação desse material na região de Jaguari, RS.

Quanto aos cultivos, foi constatada uma tendência de manutenção e mesmo aumento da produtividade de colmos da cana-planta para a cana-soca, tanto para os clones de ciclo médio-tardio quanto para os precoces (Tabela 4), a qual foi marcante para o RB008347, que duplicou a produção de colmos. Entretanto, esse clone apresentou baixa produção de colmos na cana-planta. A produtividade de colmos de 13 dos 25 clones avaliados foi maior no cultivo

Tabela 4: Produtividade $\left(\mathrm{t} \mathrm{ha}^{-1}\right)$ de clones de cana-de-açúcar de ciclos precoce e médio-tardio, de cana-planta e cana-soca. Jaguari, RS, 2009-2011 $1^{(1)}$

\begin{tabular}{|c|c|c|c|}
\hline \multicolumn{4}{|c|}{ Ciclo precoce } \\
\hline Clones & Cana-planta & Cana-soca & Total \\
\hline RB925211 & $84,90 \mathrm{bcA}$ & 108,03 abcdA & $192,90 \mathrm{bcd}$ \\
\hline RB925345 & $92,90 \mathrm{abB}$ & $122,23 \mathrm{abA}$ & $215,20 a b$ \\
\hline RB965911 & $104,73 \mathrm{aB}$ & $132,90 \mathrm{aA}$ & $237,70 \mathrm{a}$ \\
\hline RB835054 & $80,60 \mathrm{bcB}$ & $115,20 \mathrm{abcA}$ & $195,80 \mathrm{bcd}$ \\
\hline RB965902 & $79,37 \mathrm{bcB}$ & $96,83 \mathrm{bcdA}$ & $176,20 \mathrm{cdef}$ \\
\hline RB855156 & $81,20 \mathrm{bcB}$ & $130,37 \mathrm{aA}$ & $211,50 \mathrm{abc}$ \\
\hline RB966923 & $79,47 \mathrm{bcA}$ & 94,83 bcdeA & $174,30 \mathrm{def}$ \\
\hline RB986955 & $69,60 \mathrm{cB}$ & $86,00 \mathrm{de} A$ & 155,60 ef \\
\hline RB975932 & $92,03 \mathrm{abA}$ & 89,10 cdeA & 181,10 bcde \\
\hline RB935581 & $80,27 \mathrm{bcA}$ & $83,80 \mathrm{de} A$ & $164,00 \mathrm{def}$ \\
\hline RB996961 & $73,70 \mathrm{cA}$ & $87,43 \mathrm{cde} A$ & $161,10 \mathrm{def}$ \\
\hline RB986419 & $75,80 \mathrm{bcA}$ & $67,83 \mathrm{eA}$ & $143,60 \mathrm{f}$ \\
\hline Média & 82,88 & 101,21 & 184,10 \\
\hline CV $(\%)$ & 7,19 & 9,24 & 6,52 \\
\hline \multicolumn{4}{|c|}{ Ciclo médio-tardio } \\
\hline Clone & Cana-planta & Cana-soca & Total \\
\hline RB975038 & $81,90 \mathrm{bcdB}$ & $103,20 \mathrm{bcdA}$ & $185,20 \mathrm{bcd}$ \\
\hline RB975019 & $104,50 \mathrm{abcB}$ & $132,90 \mathrm{abA}$ & $237,50 \mathrm{ab}$ \\
\hline RB987935 & $118,20 \mathrm{aA}$ & $134,30 \mathrm{abA}$ & $252,50 \mathrm{a}$ \\
\hline RB72454 & $102,80 \mathrm{abcA}$ & $86,90 \mathrm{dA}$ & $189,70 \mathrm{bcd}$ \\
\hline RB845210 & $82,30 \mathrm{bcdB}$ & $140,50 \mathrm{aA}$ & $222,80 \mathrm{abc}$ \\
\hline RB925268 & 86,90 abcdB & 115,60 abcdA & $202,60 \mathrm{abcd}$ \\
\hline RB867515 & $110,20 \mathrm{abcA}$ & $125,90 \mathrm{abcA}$ & $236,20 a b c$ \\
\hline RB966229 & 85,10 abcdA & $81,80 \mathrm{dA}$ & $167,00 \mathrm{~cd}$ \\
\hline RB935744 & $114,80 \mathrm{abA}$ & 115,50 abcdA & $230,40 \mathrm{abc}$ \\
\hline RB947625 & 89,10 abcdA & $88,40 \mathrm{dA}$ & $177,50 \mathrm{bcd}$ \\
\hline RB945177 & $56,90 \mathrm{~dB}$ & $95,60 \mathrm{cdA}$ & $152,50 \quad d$ \\
\hline RB008347 & $60,80 \mathrm{~dB}$ & $125,70 \mathrm{abcA}$ & $186,50 \mathrm{bcd}$ \\
\hline RB975329 & $80,40 \mathrm{cdB}$ & $133,00 \mathrm{abA}$ & $213,50 \mathrm{abc}$ \\
\hline Média & 90,30 & 113,80 & 204,20 \\
\hline$\overline{\mathrm{CV}}(\%)$ & 12,73 & 10,39 & 9,88 \\
\hline
\end{tabular}

${ }^{(1)}$ Médias seguidas pela mesma letra minúscula na coluna e maiúscula na linha, não diferem entre si pelo teste de Scott Knott , a 5\% de probabilidade de erro. 
de cana-soca do que no cultivo de cana-planta. Hanauer (2011), trabalhando com três clones de cana-de-açúcar, verificou maior produtividade em cana-soca somente com o clone SP711406, sendo que os clones IAC822045 e CB4176 apresentaram mesma produção nos dois cultivos. Aude et al. (1992) também verificaram maior produtividade de colmos em cana-soca, corroborando os resultados encontrados neste estudo para alguns clones de ciclos precoce e médio-tardio.

Independentemente do cultivo, entre os clones que se salientaram pela produtividade de colmos (Tabela 4) encontram-se RB925345, RB965911, RB935744, RB925268, RB975019 e RB987935, os quais também foram os mais produtivos em trabalho realizado por Harter et al. (2011), em Pelotas, RS. A produtividade desses clones situou-se próxima ou acima de $100 \mathrm{t} \mathrm{ha}^{-1}$, valor similar ao encontrado em regiões tradicionais de produção de cana-de-açúcar, como aqueles citados por Landell et al. (2004), de 103,4 até 126,6 t ha ${ }^{-1}$ de colmos industrializáveis, para Ribeirão Preto, Adamantina, Mococa/Mantiqueira e Triângulo Mineiro.

Considerando-se a produtividade acumulada de colmos nos dois cultivos, destacaram-se principalmente os clones RB965911, RB925345, RB855156, RB987935, RB935744, RB867515, RB975019, RB925268, RB845210 e RB975329

(Tabela 4), os quais apresentaram produção superior a 200 $\mathrm{t} \mathrm{ha}^{-1}$. Com base nesses resultados, pode-se concluir que, entre os clones avaliados, esses são os mais indicados para a região de Jaguari, RS. Entre os demais clones testados, existem alguns que também apresentaram potencial para utilização, uma vez que suas produtividades em cada cultivo foram muito superiores ao valor médio de $41,8 \mathrm{t} \mathrm{ha}^{-1}$, encontrado nas condições do RS, estando até mesmo acima da média nacional de 81 t ha $^{-1}$ (Conab, 2012). Portanto, vários clones mostraram excelente adaptação às condições de cultivo da região da Depressão Central do Rio Grande do Sul, pois apresentaram produtividade de colmos maior que a média estadual.

O número de colmos, seguido por altura, diâmetro e densidade são as variáveis que mais se correlacionam com a produtividade de colmos (Ferreira et al., 2007; Suguitani, 2006). Observou-se que os clones RB867515 e RB947625 apresentaram decréscimo do número de colmos, do cultivo de cana-planta para cana-soca (Tabela 3). Porém, somente o clone RB867515 destacou-se entre os de ciclo médio-tardio pela produção de colmos (Tabela 4). Essa resposta pode ser atribuída ao elevado comprimento de colmo verificado neste clone (Tabela 3 ), compensando a diminuição do número (Tabela 3), o que não ocorreu com o clone RB947625, o qual apresentou menor comprimento de colmo.

De maneira geral, a análise dos atributos de colmo evidenciou que os clones com maiores valores em três das variáveis agronômicas (número de colmos $\mathrm{m}^{-1}$, comprimento e diâmetro do colmo, principalmente as duas primeiras) foram aqueles que apresentaram elevada produtividade de colmos.

A análise global dos resultados de produtividade de colmos (Tabela 4) evidencia que existem clones, tanto de ciclo precoce quanto médio-tardio, com produções próxima ou acima da média nacional, independentemente do cultivo. Portanto, é viável a utilização de clones de diferentes ciclos, a fim de aumentar o período de produção de colmos, sem que ocorra uma redução da massa de colmos, mantendo-se produções acima de $100 \mathrm{t} \mathrm{ha}^{-1}$. Esse é um fator-chave, no estado do RS, para viabilizar a cadeia produtiva do álcool, pois atua sobre um dos "gargalos" do agronegócio da cana-de-açúcar, reduzindo seu período de entressafra.

\section{CONCLUSÕES}

A produtividade de colmos tende a ser igual ou superior, em cana-soca, comparando-se com a da cana-planta. A utilização de clones de ciclos precoce e médio-tardio com elevada produtividade permite a ampliação do período de safra, sem que ocorra queda acentuada da produção de colmos, em cana-planta e cana-soca.

Os clones RB965911, RB925345, RB855156, RB987935, RB935744, RB867515, RB975019, RB925268, RB845210e RB975329 são os mais adaptados às condições edafo-climáticas da região da Depressão Central do Rio Grande do Sul, pois apresentam maior produtividade acumulada de colmos (cana-planta e cana-soca) acima de $200 \mathrm{t} \mathrm{ha}^{-1}$.

\section{AGRADECIMENTOS}

Os autores agradecem à FINEP pelo apoio financeiro por meio do projeto número 123456, convênio 0123456789 ; ao Conselho Nacional de Desenvolvimento Científico e Tecnológico (CNPq), pela concessão de Bolsa de Iniciação Científica, e à Fundação de Amparo a Pesquisa do Estado do Rio Grande do Sul (Fapergs), assim como ao CNPq, pela Bolsa de Produtividade em Pesquisa.

\section{REFERÊNCIAS}

Aude IS, Marchezan E, Dariva T \& Pasqualetto A (1994) Avaliação de cultivares de cana-de-açúcar em Santa Maria-RS. Ciência Rural, 24:471-475.

Aude IS, Marchezan E, Pignataro IAB \& Pasqualetto A (1992) Época de plantio e seus efeitos na produtividade e teor de sólidos solúveis no caldo da cana-de-açúcar. Ciência Rural, 22:131-137

Barbieri V \& Villa Nova NA (1981) Climatologia e a cana-deaçúcar. In: Vieira MAS, Hoffmann HP, Pimenta TG, Arizono H, Basinello AJ, Oliveira FFS, Matsuoka S, Barcelos JET \& Godin PRG (Eds.) Curso intensivo sobre cana-de-açúcar. Araras, IAA/ Planalsucar. p.1-100. 
Canal IN \& Matzenbacher RS (1986) Avaliação de cultivares de cana-de-açúcar. Trigo e soja, 83:03-06.

CQFS - Comissão de Química e Fertilidade do Solo (2004) Manual de Adubação e de Calagem para os Estados do Rio Grande do Sul e de Santa Catarina. $4^{\mathrm{a}}$ ed. Porto Alegre, SBCS. 394p.

Cargnelutti Filho A, Matzenauer R \& Trindade Júlio Kuhn da (2004) Ajustes de funções de distribuição de probabilidade à radiação solar global no Estado do Rio Grande do Sul. Pesquisa Agropecuária Brasileira, Brasília, 39:1157-1166.

Conab - Companhia Nacional de Abastecimento (2012) Avaliação da safra brasileira: cana-de-açúcar. Disponível em: http:// www.conab.gov.br/OlalaCMS/uploads/arquivos/12_04_10 _09_19_04_boletim_de_cana.pdf /. Acessado em: 22 de junho de 2012 .

Fepagro - Fundação Estadual de Pesquisa Agropecuária do Estado Rio Grande do Sul (2012) Pesquisadores buscam variedades de cana-de-açúcar resistentes ao clima gaúcho. Disponível em: $<$ http://www.fepagro.rs.gov.br/conteudo/1620/?Pesquisadores_buscam_variedades_de_cana-de-a\%C3\% A 7\% C3\% BAcar_resistentes_ao_clima_ga\%C3\%BAcho>. Acesso em: 13 de novembro de 2012.

Ferreira FM, Barros WS, Silva FL, Barbosa MHP, Cruz CD \& Bastos IT (2007) Relações fenotípicas e genotípicas entre componentes de produção em cana-de-açúcar. Bragantia, 66:605610 .

Hanauer JG (2011) Crescimento, desenvolvimento e produtividade em cultivo de cana-planta e cana-soca de um ano em Santa Maria, RS. Dissertação de Mestrado. Universidade Federal de Santa Maria, Santa Maria. 81p.
Harter A, Silva SDAS \& Verissimo M (2011) Avaliação de genótipos de cana-de-açúcar em ciclo de primeira soca no município de Pelotas, RS. In: XX Congresso de Iniciação Cientifica e III Mostra Cientifica, Pelotas. Anais, CD-ROM.

Hoffmann HP (2008) Variedades e manejos varietal da cana-deaçúcar, a obtenção de novos clones. Disponível em: http:// www.etanol.ufscar.br/palestras-01-09-08/variedades-e-manejovarietal. Acessado em: 12 de maio de 2011.

Landell MGA, Campana MP, Figueiredo P, Silva MA, Vasconcelos ACM, Bidóia MAP, Xavier MA, Dinardo-Miranda LL, Prado H, Rosseto R, Santos AS, Cavichioli JC, Martins ALM, Kanthack RAD, Gallo PB, Veiga Filho AA \& Silva DN (2004) Variedades de cana-de-açúcar para o Centro-Sul do Brasil. Campinas, IAC. 33p. (Boletim Técnico, 195).

Leite RLL, Santos AC, Oliveira LBT, Araújo AS \& Neiva JNM (2010) Produção e desenvolvimento de cultivares de cana-deaçúcar em argissolo vermelho eutroférrico no estado do Tocantins. Revista Amazônia, Ciência \& Desenvolvimento, 10:181-194.

Moreno JA (1961) Clima do Rio Grande do Sul. Porto Alegre, Secretaria da Agricultura. 43p.

Rodrigues DJ (1995) Fisiologia da cana-de-açúcar. Botucatu, Unesp. 99 p.

Suguitani C (2006) Entendendo o crescimento e produção da canade-açúcar: avaliação do modelo Mosicas. Tese de doutorado. Escola Superior de Agricultura "Luiz de Queiroz", Piracicaba. $60 \mathrm{p}$. 